\title{
Propuesta de un modelo conceptual para la vigilancia del virus de inmunodeficiencia humana (VIH), enfocado en el seguimiento a las personas
}

Dra. Ingrid del Carmen Castillo Morales

Fecha de recepción: 14/1/2021

Fecha de aceptación: 18/2/2021

\section{Resumen}

El objetivo del estudio fue proponer un modelo conceptual de vigilancia epidemiológica para el virus de la inmunodeficiencia humana (VIH) basado en el seguimiento a las personas. El método aplicado fue un estudio cualitativo descriptivo, con el uso del Modelo de Sistematización de Experiencias de Óscar Jara; se recolectaron las lecciones aprendidas de los actores clave, tanto del Ministerio de Salud como del Seguro Social y agencias cooperantes en la respuesta al VIH. Se sistematizó la información de los estándares internacionales y la información obtenida de la Evaluación de la Vigilancia del VIH.

Como resultado se obtuvo la incorporación de un código único de identificación para la trazabilidad del paciente, por tanto, se identificaron tres momentos para la vigilancia; prevención que abarcará desde la primera prueba que se realiza independientemente de su resultado, el objetivo será vigilar que permanezca negativo o sea oportunamente identificado y vinculado al tratamiento. La vigilancia en el momento de la atención tiene por objetivo, adherir el paciente al tratamiento para lograr una carga viral indetectable, el cual termina en ese momento con el potencial infeccioso del paciente. Por último, los cuidados crónicos y fallecimiento que implicó la vigilancia de las infecciones oportunistas, enfermedades crónicas y mortalidad.

La investigación concluye que este modelo responde a las necesidades de la epidemia y se adapta a los sectores guatemaltecos involucrados en la respuesta, e identifica puntos clave en el registro para la elaboración de indicadores. Propone vías para incorporar y medir atributos cuantitativos de la vigilancia, puesto que abarca personas sanas para promover que continúen siendo negativas.

Palabras clave: virus de inmunodeficiencia humana, vigilancia epidemiológica, modelo conceptual, vinculación.

\footnotetext{
${ }^{1}$ Médica y cirujana, Universidad de San Carlos de Guatemala; magíster en Salud Pública con Énfasis en Epidemiología, Universidad Rafael Landívar de Guatemala.
} 


\section{Proposal for a conceptual model for human immunodeficiency virus (HIV) surveillance, focused on follow-up to the people}

\section{Abstract}

The objective of the study was to propose a conceptual model of epidemiological surveillance for the human immunodeficiency virus (HIV) based on the follow-up of individuals. The method applied was a descriptive qualitative study, using Oscar Jara's Experience Systematization Model; lessons learned were collected from key actors, both from the Ministry of Health and Social Security and cooperating agencies in the response to HIV. The information from international standards and the information obtained from the HIV Surveillance Evaluation was systematized.

As a result, the incorporation of a unique identification code for patient traceability was obtained, therefore, three moments for surveillance were identified; prevention that will cover from the first test that is performed regardless of its result, the objective will be to monitor that the patient remains negative or is identified in a timely manner and linked to treatment. Surveillance at the time of care is aimed at adhering the patient to treatment to achieve an undetectable viral load, which ends at that moment with the patient's infectious potential. Finally, chronic care and death involved surveillance of opportunistic infections, chronic diseases and mortality.

The research concludes that this model responds to the needs of the epidemic and is adapted to the Guatemalan sectors involved in the response, and identifies key points in the registry for the development of indicators. It proposes ways to incorporate and measure quantitative attributes of surveillance, as it covers healthy people to promote continued negativity.

Key words: human immunodeficiency virus, epidemiological surveillance, conceptual model, linkage

\section{Introducción}

A principios de la década de los ochenta, al inicio de la epidemia del virus de la inmunodeficiencia humana $(\mathrm{VIH})$ en Guatemala, la única posibilidad de identificar los casos era mediante la historia clínica, esto significa que los casos se detectaban y notificaban en fase avanzada o fase de síndrome de inmunodeficiencia adquirida (sida). Según el Departamento de Epidemiología del Ministerio de Salud Pública y Asistencia Social, a medida que la tecnología diagnóstica lo permitió, la vigilancia epidemiológica desplaza la definición de "Caso SIDA», por la definición de "Caso por $\mathrm{VIH}$ ». Así mismo, la implementación de la Terapia Antirretroviral permitió que la expectativa de vida de los pacientes diagnosticados con VIH se ampliara. Los cambios en la historia natural de la enfermedad producen una demanda específica en el sistema de información del Ministerio de Salud y son la razón primordial de este estudio.

El modelo propuesto en esta investigación se encuentra basado en los resultados de la última evaluación del Sistema de Vigilancia de $\mathrm{VIH}$, la revisión de los estándares internacionales y la sistematización de experiencias de expertos, cuya principal característica es dar seguimiento a las personas. Este modelo integra las particularidades de la vigilancia de la transmisión materno infantil, la mortalidad, la vigilancia de segunda generación, entre otros; busca involucrar a todas las instituciones del sector salud que trabajan para detener la epidemia. 


\section{Método}

Se realizó un estudio cualitativo descriptivo con la aplicación del Modelo de Sistematización de Experiencias de Óscar Jara, para lo cual fueron recolectadas las lecciones aprendidas de los actores clave del Ministerio de Salud, Seguro Social y agencias cooperantes relacionadas al VIH. Se sistematizó la información de los estándares internacionales y la información obtenida de la evaluación de la vigilancia. El proceso parte del análisis teórico y modélico, así como de la estandarización de la información obtenida, lo cual permitió estructurar un modelo específico. Se observan las tres fases siguientes:

Fase I. Revisión bibliográfica y organización de la información:

Se realizó una revisión bibliográfica exhaustiva, con la finalidad de obtener los lineamientos e identificar los elementos para el seguimiento a las personas.

Fase II. Entrevista a informantes clave:

Se efectuó una entrevista dirigida a actores clave involucrados en la vigilancia epidemiológica y respuesta del $\mathrm{VIH}$, tanto a nivel central como en los servicios de salud. También se contó con la participación de actores del Programa Conjunto de las Naciones Unidas para el VIH (Onusida), del Centro para el Control y Prevención de Enfermedades (CDC) y de la Agencia de los Estados Unidos para el Desarrollo (Usaid). La entrevista tuvo una duración en promedio de treinta y cinco minutos, su objetivo era conocer las dificultades vigentes para acceder a la información oportuna y generar la toma de decisiones. Las entrevistas fueron transcritas para su sistematización y análisis; de esta forma se identificaron las ideas más relevantes o en las que coincidían los actores, con el objetivo de elaborar el libro de códigos. Se introdujeron en el programa Dedoose para su codificación y posterior análisis.

Fase III. Estructuración del nuevo modelo de vigilancia:

Se estructuró el Protocolo de Vigilancia Epidemiológica de VIH basado en los hallazgos de las fases I y II y con ello abarcar todos los aspectos que la epidemia de $\mathrm{VIH}$ requiere; parte de un registro apropiado de los datos según el nivel en que se encuentra la atención para plantear la ruta y convertir estos datos en información nacional y local de VIH.

\section{Análisis de datos}

Fase I: la vigilancia epidemiológica debe adaptarse al evento en salud que se desea vigilar y abarca las intervenciones programadas, de forma que pueda extraerse de esta información el estado de la epidemia y el impacto de las intervenciones en esta. Debido al análisis de un evento cuya particularidad es ser una enfermedad transmisible y crónica, que pierde y recupera su potencial infeccioso según la intervención aplicada y la temporalidad en que se aplicó; se identificaron tres momentos en la historia natural de la misma, que poseen características propias y demandan intervenciones específicas. 
1. El primer momento es la fase que abarca desde el inicio de la vida sexual hasta que se tiene conocimiento del estatus serológico. Si este es negativo la persona permanece en este primer momento; si es positivo pasa a la siguiente fase.

2. Fase en la cual la persona conoce su estado serológico positivo.

3. Fase de la enfermedad crónica.

Fase II: la vigilancia epidemiológica puede ser resumida en la frase «información para la acción». Los atributos de la vigilancia son una serie de características necesarias para que la información esté disponible y organizada de forma adecuada y con ello se puedan realizar las intervenciones. Se realizaron entrevistas a informantes clave del Ministerio de Salud Pública y Asistencia Social (MSPAS), del Instituto Guatemalteco de Seguridad Social (IGSS) y de organizaciones cooperantes como el Programa Conjunto de las Naciones Unidas sobre el VIH/sida, el Centro para el Control y la Prevención de Enfermedades (CDC) y la Agencia de los Estados Unidos para el Desarrollo Internacional (Usaid).

Fase III: con los hallazgos de las fases I y II se estructuraron procesos inmediatos para la operativización de la vigilancia epidemiológica, lo que requiere establecer el siguiente algoritmo para el registro de personas positivas o negativas para VIH en el sistema de vigilancia epidemiológica que se presentan en los esquemas 2 y 3.

\section{Resultados y discusión}

\section{Fase 1}

Figura 1. Etapas de la vigilancia del VIH
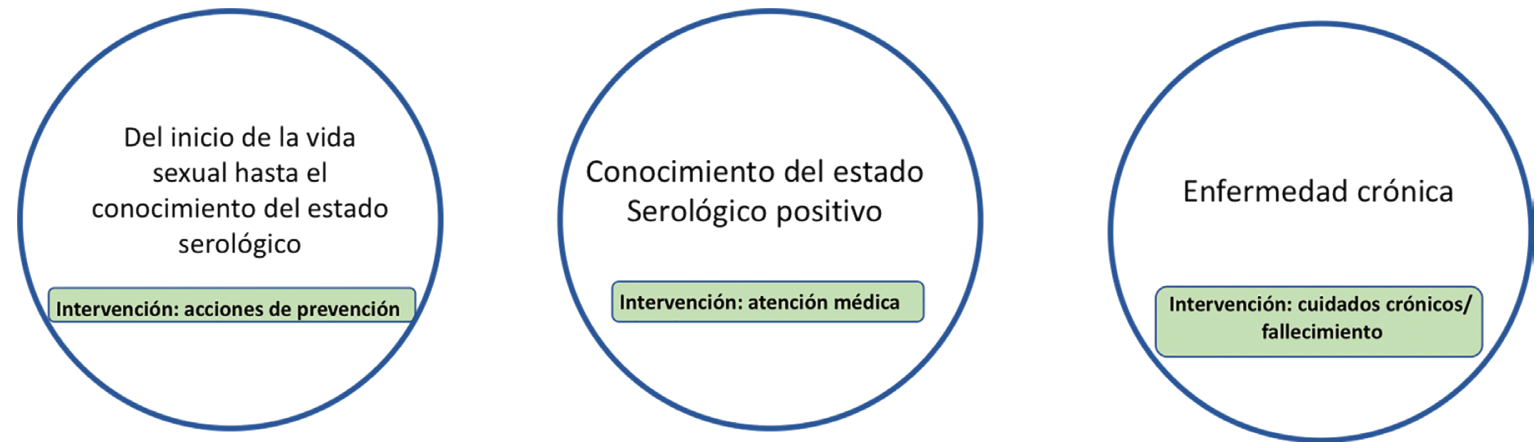

Fuente: elaboración propia con base en el resutado del análisis de entrevistas y revisión bibliográfica.

La figura 1 identifica tres momentos para la vigilancia:

1. Prevención: tiene el propósito de conocer el estado serológico de las personas para poder tomar decisiones a partir de este conocimiento. Si la persona es negativa, se promueve un estilo de vida y la tecnología necesaria para que continúe siendo VIH negativa; si es positiva, para que pueda ser vinculada a un servicio de salud para iniciar tratamiento.

2. Atención: en esta etapa es importante vigilar tres momentos, la vinculación, es decir, el momento cuando el paciente asiste a un servicio de salud para obtener atención integral; el inicio de tratamiento, en el cual es deseable conocer si presenta alguna clase de resistencia a antirretrovirales, de forma que en este punto inicie la fármaco vigilancia, es decir, que es necesario obtener la fecha en la que inició tratamiento (TAR) y conocer la sensibilidad que presenta a este y, la carga viral suprimida, es decir, la fecha en que el recuento viral está por debajo de $50 \mathrm{c} / \mathrm{ml}$. 
3. Cuidados crónicos y fallecimiento: en este estadio se necesitan vigilar los casos en dos escenarios. El primero es en aquellos, cuyo estado serológico es conocido y tuvieron acceso a atención y tratamiento, por tanto, para fines de vigilancia, será necesario conocer cuántas personas han tenido enfermedades crónicas, causadas o no por el tratamiento. El segundo se vincula a los pacientes que no fueron diagnosticados oportunamente, por consiguiente, pueden presentar enfermedades oportunistas. Para ambos casos es indispensable la vigilancia de la mortalidad, pero según información proporcionada por el Departamento de Epidemiología del MSPAS es un proceso que hasta hoy no se realiza; la única fuente vinculada a la mortalidad son los registros del Instituto Nacional de Estadística (INE) que datan del 2005.

\section{Fase II}

Luego de clasificar y ordenar la información obtenida de las entrevistas se encontraron los siguientes resultados:

- Dificultad para acceder a la información. Algunos actores que participan de forma activa en el registro de datos, no pueden acceder a la información sobre $\mathrm{VIH}$. Retroalimentación. Quienes logran acceder a ella afirman que la vía para conseguirla no es amigable y

es confusa. La información que se genera a través de la vigilancia epidemiológica de VIH no llega a ser del conocimiento de todos los servicios de salud y cuando llega la periodicidad no es la adecuada para la toma de decisiones. Se solicita que sea en tiempo real.

- Complejidad. Los instrumentos nacionales para el registro, son confusos para su uso en el registro, al igual que el acceso a la plataforma del sistema de información.

- Calidad del dato. La información generada por la vigilancia epidemiológica no es verificable, evidencia subregistro o registro incompleto.

- Acceso a la base de datos. Se manifestó interés general en tener la base de datos para análisis propios de cada sector.

- Personal insuficiente. La falta de personal fue una respuesta unánime entre los entrevistados. Afirman que el trabajo que hay que realizar demanda un equipo que se dedique al evento.

- Uso de la información. La información generada por la vigilancia epidemiológica es utilizada por los actores del MSPAS para hacer informes, sin embargo, las instituciones ajenas a este Ministerio no utilizan los datos generados, debido a las características y su calidad.

- Coordinación entre los sectores. Es necesario mejorar la comunicación del Departamento de Epidemiología a nivel central hacia los distritos de salud y entre las instituciones para la generación de información a nivel nacional. 


\begin{tabular}{|c|c|c|}
\hline Atributos & Modelo actual & Aplicación en el nuevo modelo \\
\hline SIMPLICIDAD & $\begin{array}{l}\text { Múltiples instrumentos de } \\
\text { registro con duplicidad de } \\
\text { funciones, registrados en varias } \\
\text { plataformas. }\end{array}$ & $\begin{array}{l}\text { Presencia de } 4 \text { herramientas para el registro } \\
\text { (prevención, diagnóstico, tratamiento/ } \\
\text { atención integral, mortalidad) en un único } \\
\text { sistema de información. }\end{array}$ \\
\hline FLEXIBILIDAD & $\begin{array}{l}\text { La última evaluación realizada } \\
\text { fue en el } 2013 .\end{array}$ & $\begin{array}{l}\text { Evaluación cada } 3 \text { años para actualización e } \\
\text { implementación de cambios. }\end{array}$ \\
\hline CALIDAD DEL DATO & $\begin{array}{l}\text { No existe mecanismo } \\
\text { actualmente. }\end{array}$ & $\begin{array}{l}\text { Uso de CUI/DPI como identificador único, } \\
\text { monitoreo periódico de la vigilancia, } \\
\text { mesa intersectorial para la elaboración de } \\
\text { informes. Uso de un mismo instrumento } \\
\text { para el registro, con usuarios para todos los } \\
\text { sectores. }\end{array}$ \\
\hline
\end{tabular}

\section{ACEPTABILIDAD}

VALOR PREDICTIVO

\section{POSITIVO}

\section{REPRESENTATIVIDAD}

OPORTUNIDAD

ESTABILIDAD

UTILIDAD
No existe mecanismo actualmente.
Capacitación continua a través de una plataforma virtual. Establecer canales de comunicación. Retroalimentación mensual a los distritos/sectores involucrados.

Se registra únicamente del caso confirmado.

No es posible medir esto actualmente.
Registro del paciente desde que se realiza la primera prueba, independientemente de su resultado.
Registro apropiado de las pruebas de forma nominal. Uso de un mismo instrumento para el registro, con usuarios para todos los sectores.

Se incluyen variables para caracterizar a poblaciones clave. Se implementa vigilancia de la transmisión materno infantil.
A pesar de que la epidemia es concentrada, la vigilancia no puede caracterizar la epidemia en poblaciones clave por no tener incluida esa variable. No se tiene información de niños expuestos a $\mathrm{VIH}$. En relación a las embarazadas no se tiene un sistema nominal y no se distinguen personas de pruebas realizadas.
Vigilancia pasiva universal. El $40 \%$ de los pacientes diagnosticados se encuentra en etapa avanzada.
Vigilancia activa universal. Se implementará vigilancia del paciente negativo.
No existe mecanismo actualmente.

No existe mecanismo actualmente.
Creación de tableros de mando que permitan dar acceso a la información de forma actualizada y disponible a todo nivel.

Fuente: elaboración propia con base en el resutado del análisis de entrevistas y revisión bibliográfica. 
Fase III

Figura 2. Registro de personas para la Vigilancia Epidemiológica

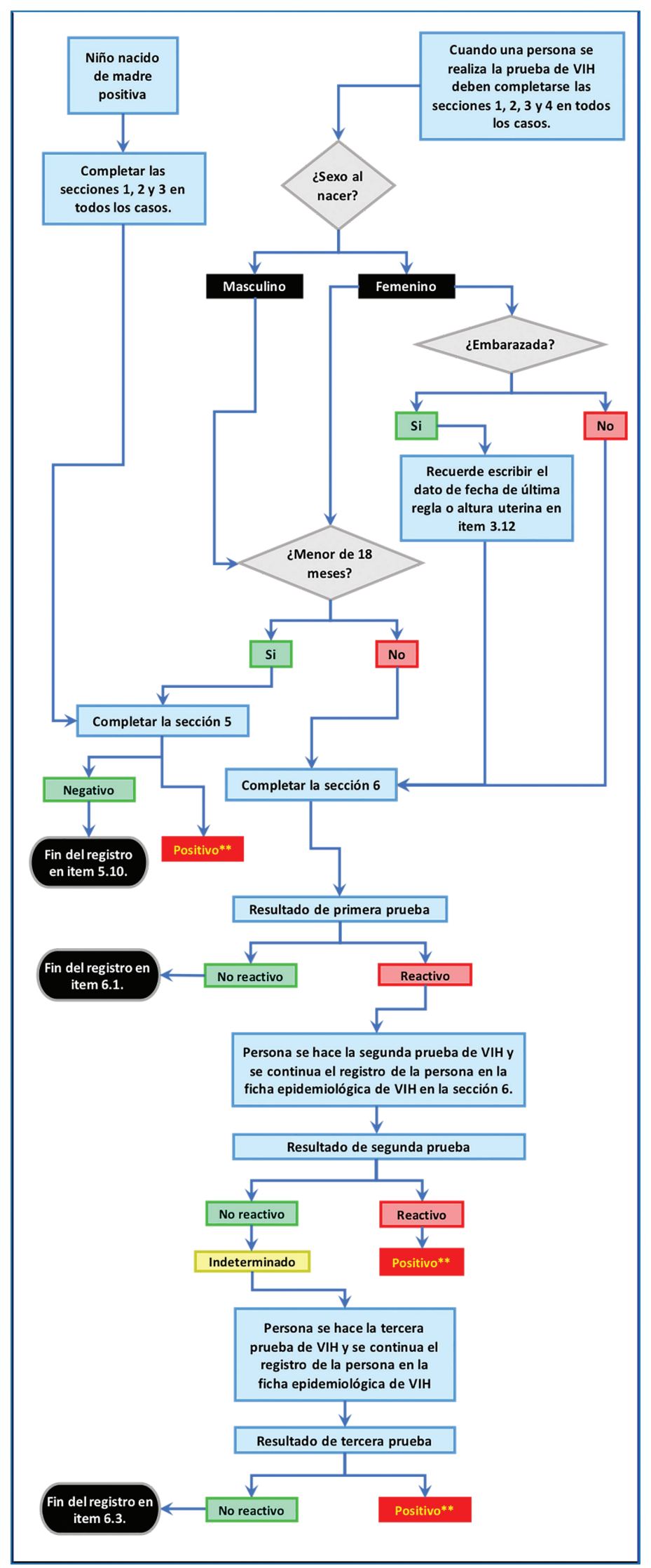

Fuente: elaboración propia con base en el resutado del análisis de entrevistas y revisión bibliográfica.
Figura 3. Registro de personas con diagnóstico positivo para la Vigilancia Epidemiológica

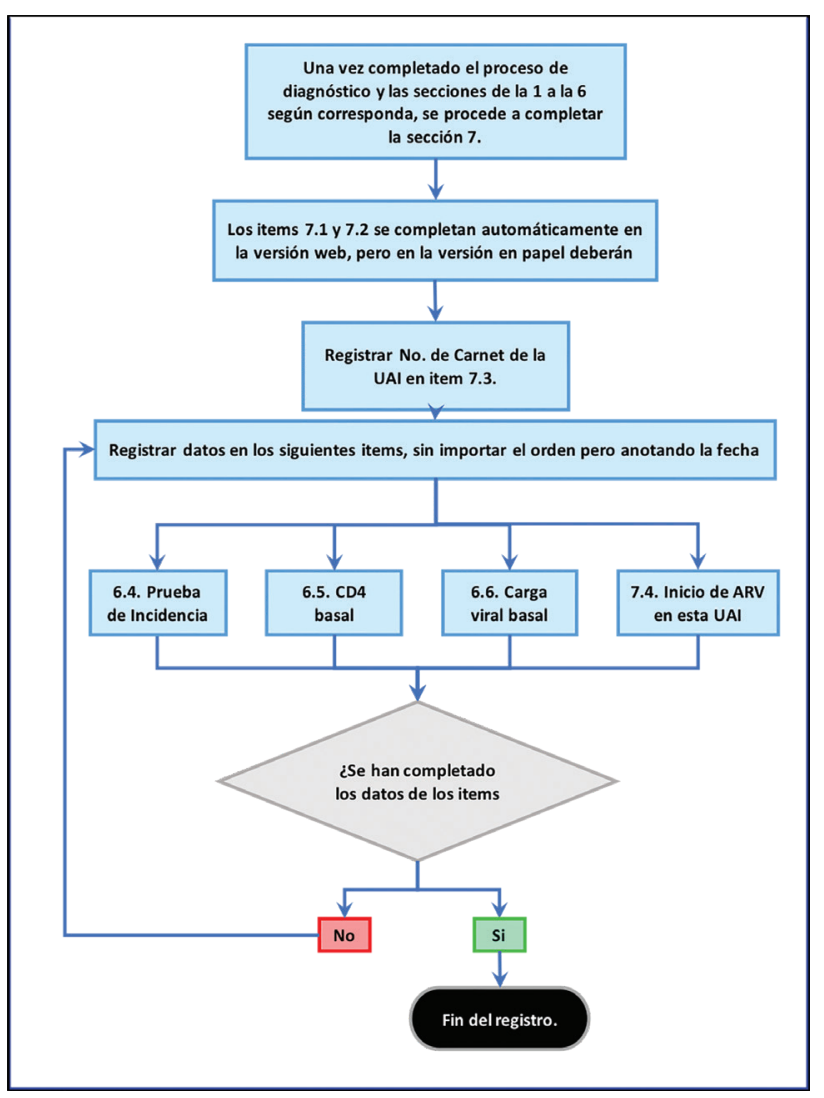

Fuente: elaboración propia con base en el resutado del análisis de entrevistas y revisión bibliográfica. 


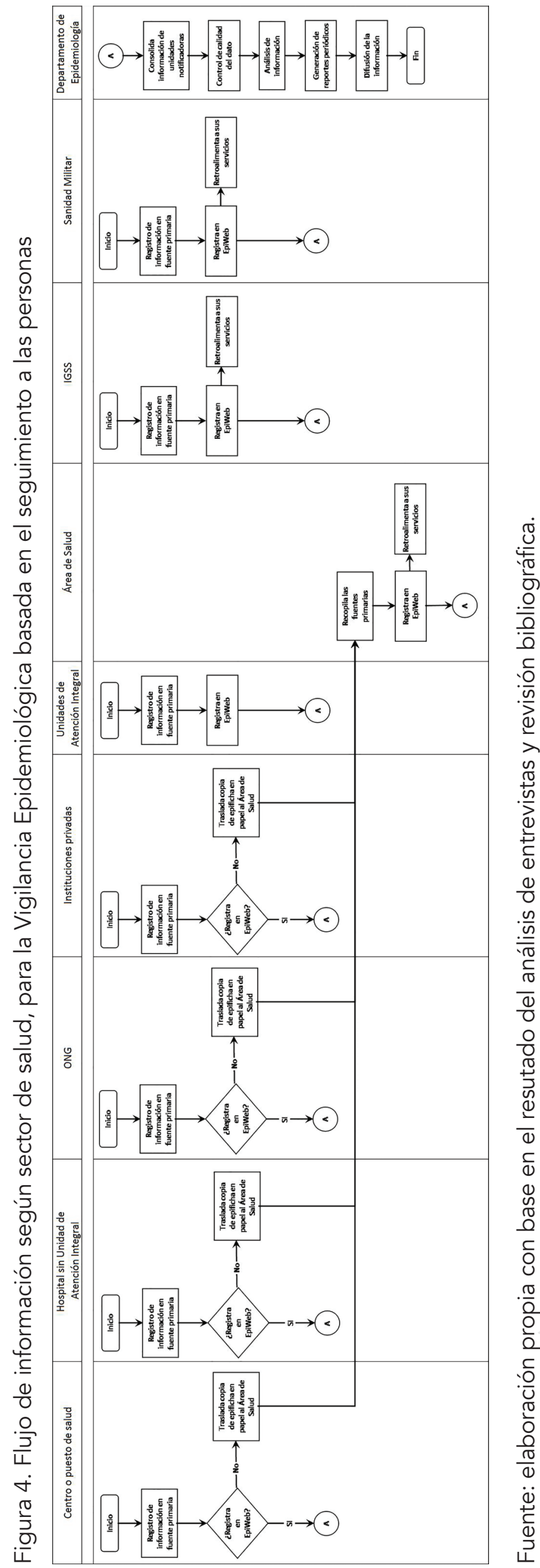

Respecto al flujo de información es necesario, para este nuevo modelo conceptual, incluir a todos los sectores que trabajan en la respuesta a la epidemia del VIH, con el fin de lograr representatividad nacional en el dato oficial, proporcionado por el Ministerio de Salud Pública y Asistencia Social.

La Vigilancia del VIH basada en el seguimiento a las personas iniciará el registro con aquel que se realice la prueba. Se tendrán, a partir de este punto, una de dos posibles rutas, la de la prevención combinada y la de la atención oportuna. Los registros de los pacientes captados en etapa de prevención serán informados a través de indicadores de la Cascada de Prevención.

Propone la búsqueda activa de casos para poder identificar al $95 \%$ de los positivos $y$, poder establecer trazabilidad de ellos a través del continuo de la atención. Realiza la vigilancia activa de los pacientes adheridos al tratamiento, para identificar infecciones oportunistas, necesi-dades de cuidados crónicos y vigilancia de la mortalidad.

Se implementa la vigilancia de la transmisión materno infantil y la vigilancia perinatal, por lo cual se incluye en la definición de caso «niño expuesto» y trazan, por tanto, una ruta para atender estos casos hasta confirmar y descartar su diagnóstico.

La vigilancia de segunda generación busca registrar aquellas conductas de riesgo, que son más frecuentes en la población y que ayudarán a reforzar las intervenciones en prevención.

La identificación de comorbilidades como infecciones oportunistas en pacientes de reciente diagnóstico, coinfección tuberculosis/ $\mathrm{VIH}$ y vigilancia de la mortalidad, no implementadas hasta hoy, tendrán un soporte normativo dentro del Protocolo de Vigilancia Epidemiológica y un instrumento para su registro.

Este modelo se propone para iniciar cambios en el sistema de información del VIH. 
Figura 5. Modelo para la Vigilancia del VIH enfocado en el seguimiento a las personas

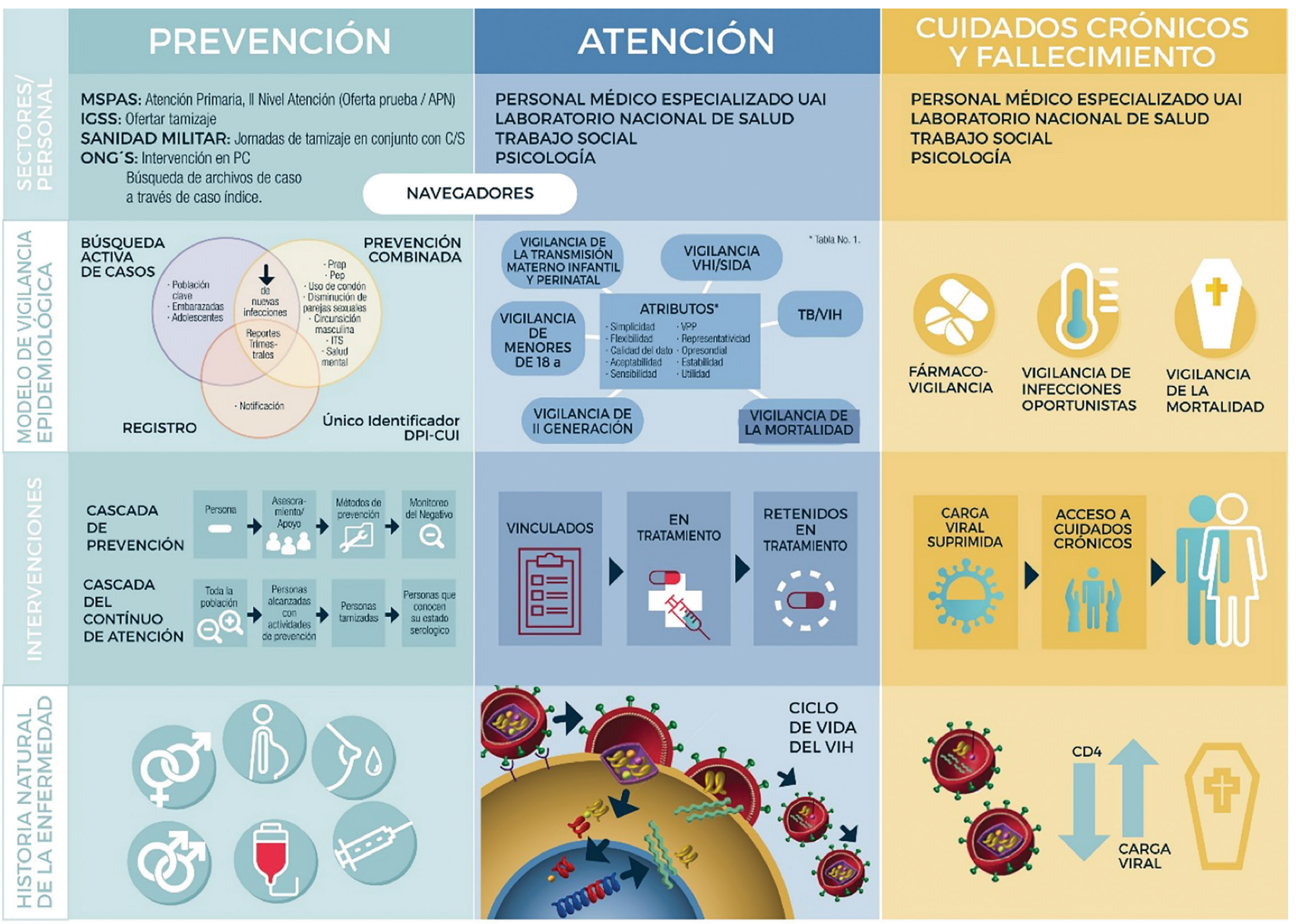

\section{Modelo para la Vigilancia del Virus de Inmunodeficiencia Humana, VIH enfocado en el seguimiento de personas}

Fuente: elaboración propia con base en los resultados de productos de la investigación.

\section{Conclusiones}

- El Modelo Conceptual de Vigilancia Epidemiológica basado en el seguimiento a las personas responde a las necesidades de la epidemia del VIH su diseño incluirá a los sectores públicos, Seguro Social (IGSS), sector privado, Sanidad Militar y sociedad civil.

- Este Modelo identifica los puntos clave del registro, que permitirán la elaboración de indicadores.

- El Modelo propone vías para incorporar y medir atributos cuantitativos de la vigilancia (sensibilidad y valor predictivo positivo).

- La Vigilancia Epidemiológica Mejorada debe abarcar a personas sanas para promover que continúen siendo negativas.

- La Vigilancia Epidemiológica Mejorada incorpora el Código Único de Identificación del Documento Personal de Identidad (CUI-DPI), para lograr trazabilidad del paciente en el registro. 


\section{Referencias}

1. OPS/OMS. Marco de Monitoreo del Continuo de la Atención al VIH. Anexo al Inf Reun: Consult Reg en América Lat y el Caribe sobre Inf epidemiológica la Infecc por el VIH. 2014;42.

2. OMS. Proyecto de Estrategia Mundial del Sector de la Salud contra el VIH para 2016-2021 [Internet]. 2016. pp. 1-17. Disponible en: https://www.who. int/reproductivehealth/GHSS_HIV_SP_06012016.pdf?ua=1

3. $\mathrm{CDC} C$ for disease control and prevention. Principios de Epidemiología. Segunda ed. Salud IN de, editor.

4. OPS/OMS Guatemala - Sistemas de Información, Vigilancia, Prevención y Control de los Riesgos a la Salud. [Internet]. [cited 2018 Aug 15]. Disponible en: https://www.paho.org/gut/index.php?option=com_content\&view=artic le\&id=428:sistemas-de-informacion-vigilancia-prevencion-y-control-de-losriesgos-a-la-salud\&Itemid $=405$

5. CDC. Normas actualizadas para la evaluación de sistemas de vigilancia en salud pública, julio 2001.

6. Salud M de SP y ASOP de la/Oficina R para CA y P de los $C$ de $C$ y $P$ de E de EU. Normas y Procedimientos del Sistema Nacional de Vigilancia Epidemiológica de Guatemala. In, pp. 1-4. Disponible en: http://epidemiologia. mspas.gob.gt/files/Protocolos\%20Antiguos/SINAVE_MSPAS.pdf

7. Onusida/OMS. Guías Prácticas para poner en práctica la vigilancia de segunda generación; 2002. Disponible en: https://data.unaids.org/ publications/irc-pub02/jc742-initiatingsgs_es.pdf

8. Regional C. Consulta Regional en América Latina y el Caribe. 2012;7-9.

9. Jara, O. Para sistematizar experiencias. San José, Costa Rica: Centro de Estudios y Publicaciones Alforja; 1998.

10. Informe Final de la Evaluación de Sistema de Vigilancia Epidemiológica del VIH en Guatemala. Guatemala: Ministerio de Salud Pública y Asistencia Social; 2013.

11. Directrices unificadas sobre prevención, diagnóstico, tratamiento y atención de la infección por el VIH para grupos de población clave. Julio del 2016. ISBN: 978-92-75-32007-5, Disponible en: https://iris. paho.org/bitstream/handle/10665.2/49094/9789275320075_spa. pdf? sequence $=1 \&$ isAllowed $=y \& u a=1$

12. Consolidated guidelines on person-centred HIV patient monitoring and case surveillance ISBN 978-92-4-151263-3, Disponible en: http:// apps.who.int/iris/bitstream/handle/10665/255702/9789241512633-eng. pdf;jsessionid=FFD7810EBCBD1DAA02A4AC6EBE46E110? sequence $=1$

13. Departamento de Epidemiología, Ministerio de Salud Pública y Asistencia Social. Protocolo de Vigilancia Epidemiológica de VIH. Guatemala. 2018. 